\title{
Motion analysis of thumb in cellular phone use
}

\author{
Naotaka Sakai* and Satoshi Shimawaki \\ Department of Mechanical and Intelligent Engineering, Utsunomiya University, 7-1-2 Yoto, Utsunomiya 321-8585, Japan
}

(Received 13 February 2009; final version received 9 July 2009)

\begin{abstract}
The thumb motion of 10 normal subjects during cellular phone use was measured using a reflective marker detection system to compare the maximum, minimum and range of flexion angles of the interphalangeal (IP), metacarpophalangeal (MP) and carpometacarpal (CM) joints. Two micro-reflective markers $3 \mathrm{~mm}$ in diameter were each placed on the dorsal surface of the distal phalanx, basal phalanx and metacarpal bone of the thumb. Three markers were placed on the dorsal hand in order to define the dorsal hand plane. Each subject pushed the 12 keys of a folding cellular phone with an 85-mm-long and 40-mm-wide keypad, sequentially from ' 1 ' to ' $\#$ ', and the pushing motion was recorded by six infrared video cameras for 12 seconds, using the VICON 612 system. The mean maximum flexion angle of the MP joint was significantly $(p<.05)$ larger than the CM joint, and the mean minimum flexion angle of the CM joint was significantly $(p<.01)$ smaller than the IP and MP joints. The mean range of motion of the IP joint was significantly $(p<.05)$ larger than the MP and the CM joints. In a comparison of different key-pushing motions, only the CM joint was significantly $(p<.05)$ larger in its range of motion. In conclusion, thumb motion on pushing the keys of the cellular phone was produced mainly by the MP and the CM joints. In addition, the ability to reach keys in different areas of the cellular phone keypad is regulated by changing the flexion angle of the CM joint.
\end{abstract}

Keywords: motion analysis; finger; hand; cellular phone; mobile phone

\section{Introduction}

The cellular phone has brought about a revolutionary change in human communication, and the ongoing development of hand-held micro-mobile computers is certain to cause further change in daily life. Discussion of medical issues related to cellular phones, however, has focused on the risk of use while driving and on the risk of exposure to the electromagnetic waves they emit (Jokela et al. 2004; White et al. 2004; Johal et al. 2005; Eliyahu et al. 2006); there are few references in the literature to hand motion during cellular phone use. Yoong (2005) investigated tenosynovitis in school children who were sending more than 100 text messages per day, and pointed out that a younger than expected group of patients was diagnosed with disorders related to mobile phone use, such as de Quervain's tenosynovitis. The hand motion is quite specific, in that almost all key-pushing movements involve only the thumb, while the other fingers hold the body of the cellular phone. Storr et al. (2007) reported a female patient with de Quervain's tensynovitis from cell phone use, who sent 2,500 texts per month, and stated that the specific thumb movement would cause the tenosynovitis or other overuse trouble around the thumb.

Hand motion has already been analysed using sophisticated methodology such as electrical goniometers or electromyograms (Darling and Cole 1990; Burgar et al. 1997; $\mathrm{Li}$ et al. 2000). Some recent reports have focused on
3-D motion analysis systems with reflective surface markers, including the author's analysis of finger motion during piano-playing and of the myelopathy hand from the cervical myelopathy (An et al. 1991; Sakai et al. 1996, 2006; Rash et al. 1999; Su et al. 2002; Kuo et al. 2003; Sakai 2005). Su et al. (2003) describe the accuracy and the reliability of this newly developed 3-D analysis.

In this study, we analysed hand motion during cellular phone use, concentrating on the movements of the thumb, on the hypothesis that the thumb motion would not only be busy, but also it would have a specific motion pattern during pushing keys, which could cause the tenosynovitis including de Quervain's disease. In order to clear out this point, we calculated the flexion angle of the thumb joints, and investigated the specificity of the thumb motion when pushing the keys of the cellular phone.

\section{Methods}

Ten normal male subjects with no history of trauma or disease in their hands took part in this study. Their mean age was 22.2 years, ranging from 21 to 25 , and their mean height and weight were $175.5 \mathrm{~cm}$ and $64.7 \mathrm{~kg}$. Proper informed consent was obtained from each subject.

Nine micro-reflective markers $3 \mathrm{~mm}$ in diameter were placed on the dorsal surface of the thumb and the hand,

*Corresponding author. Email: naosakai@cc.utsunomiya-u.ac.jp 


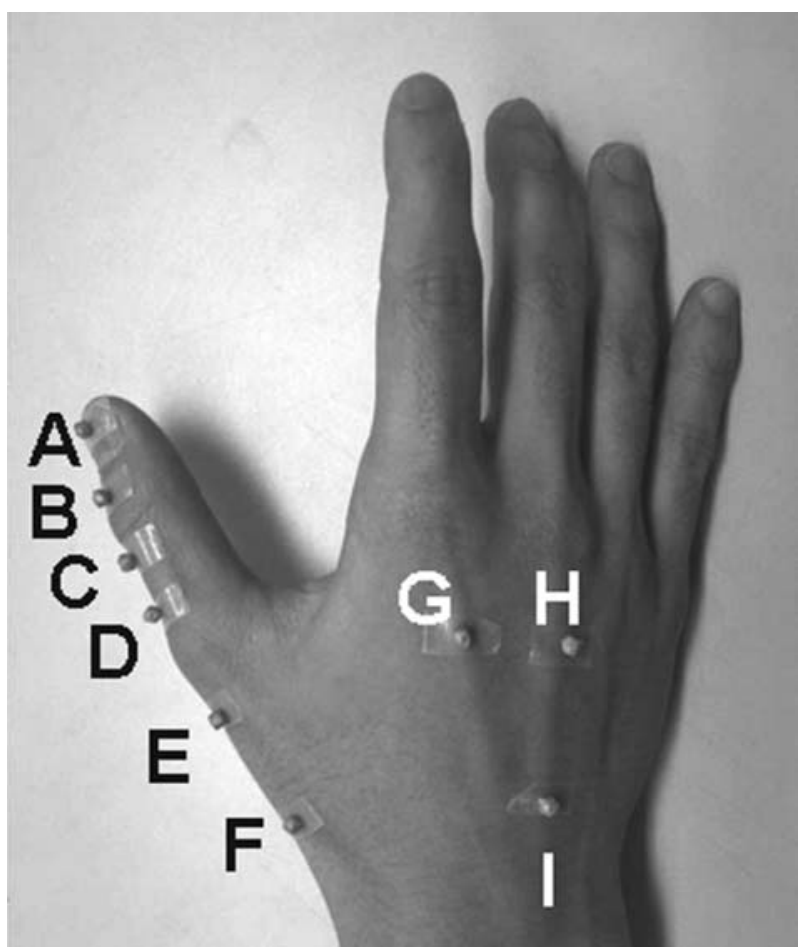

Figure 1. Nine micro-reflective markers $3 \mathrm{~mm}$ in diameter were placed on the dorsal surface of the thumb and the hand, spaced $10.4 \mathrm{~mm}$ apart.

spaced $10.4 \mathrm{~mm}$ apart (Figure 1). Two markers each were placed on the dorsal side of the distal phalanx, basal phalanx and metacarpal bone of the thumb (Figure 1: Markers A-F). Three markers were used to define the dorsal hand plane; Markers $\mathrm{G}$ and $\mathrm{H}$ were placed on the dorsal side of the second and third metacarpal heads, and Marker I on the dorsal side of the proximal second metacarpal bone.

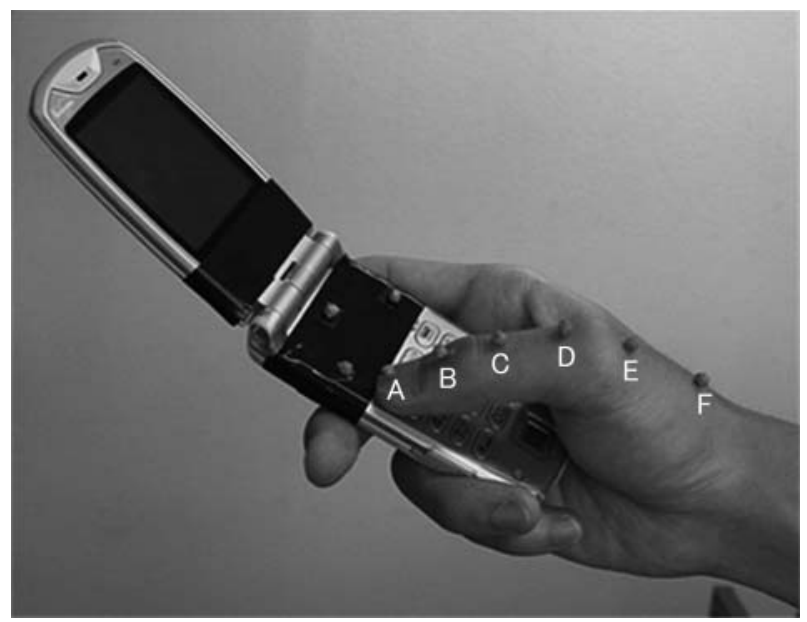

Figure 2. A folding cellular phone (C5001T, Toshiba Co.), with an $85 \mathrm{~mm}$ long and $40 \mathrm{~mm}$ wide keypad, was used for this study.
A folding cellular phone (C5001T, Toshiba Co.), with an $85 \mathrm{~mm}$ long and $40 \mathrm{~mm}$ wide keypad, was used for this study (Figure 2). Each subject was asked to hold the cellular phone in his right hand and push the 12 keys sequentially from ' 1 ' to '\#' with his right thumb. This pushing motion was recorded by six infrared video cameras for 12 seconds each, and the movements of each marker were tracked in three dimensions using the VICON 612 system (Oxford Metrics Co. Ltd., UK).

The flexion angle of the interphalangeal (IP) joint of the thumb was calculated as the angle between the vector joining Markers A and B and the vector joining Markers $\mathrm{C}$ and D. Similarly, the flexion angle of the first metacarpophalangeal (MP) joint was calculated as the angle between the vector $\mathrm{CD}$ and the vector $\mathrm{EF}$. The flexion angle of the first carpometacarpal (CM) joint was calculated as the angle between the vector EF and the plane made by Markers G, H and I, therefore, the angle between the vector DF and its projection on the plane defined by Markers G, H and I.

The maximum and minimum angles, and the arc of motion of flexion (the difference between the maximum and the minimum flexion angles) were calculated in the first IP, MP and CM joints on pushing keys. The mean maximum and minimum angles, and the range of motion of flexion were compared among the IP, MP and CM joints with Scheffe's test. In addition, the mean range of motion was compared among the ' 1 ', ' 3 ', ' $\#$ ' and '*' keys in order to investigate the difference in flexion angle of each joint of the thumb when pushing different cellular phone keys.

\section{Results}

The mean maximum flexion angle of the MP joint was $50.0 \pm 11.1$ degrees, which was significantly $(p<.05)$ larger than the $28.7 \pm 6.9$ degrees observed in the CM joint (Figure 3). The mean minimum flexion angle of the $\mathrm{CM}$ joint was $8.2 \pm 6.6$ degrees, which was significantly ( $p<$ .01) smaller than the $27.1 \pm 9.8$ degrees observed in the IP joint and the $30.3 \pm 12.4$ degrees in the MP joint. The mean range of motion of the IP joint was $9.6 \pm 7.9$ degrees, which was significantly $(p<.05)$ smaller than the $20.1 \pm$ 6.0 degrees observed in the MP joint and the $20.4 \pm 6.7$ degrees in the $\mathrm{CM}$ joint.

When different key-pushing motions were compared, only the CM joint showed a significant difference in the range of motion. The mean range of motion (flexion angle) of the CM joint on pushing the ' 1 ' key was $27.0 \pm 7.6$ degrees, which was significantly $(p<.05)$ larger than the $13.9 \pm 6.6$ degrees for the ' 3 ' key (Figure 4). Similarly, the mean range of motion on pushing ' 1 ' was also significantly $(p<.005)$ larger than the $10.4 \pm 7.9$ degrees on pushing '\#', and the mean range of motion on pushing '\#' was significantly $(p<.05)$ smaller than the $23.7 \pm 10.5$ degrees on pushing ' $*$ '. 


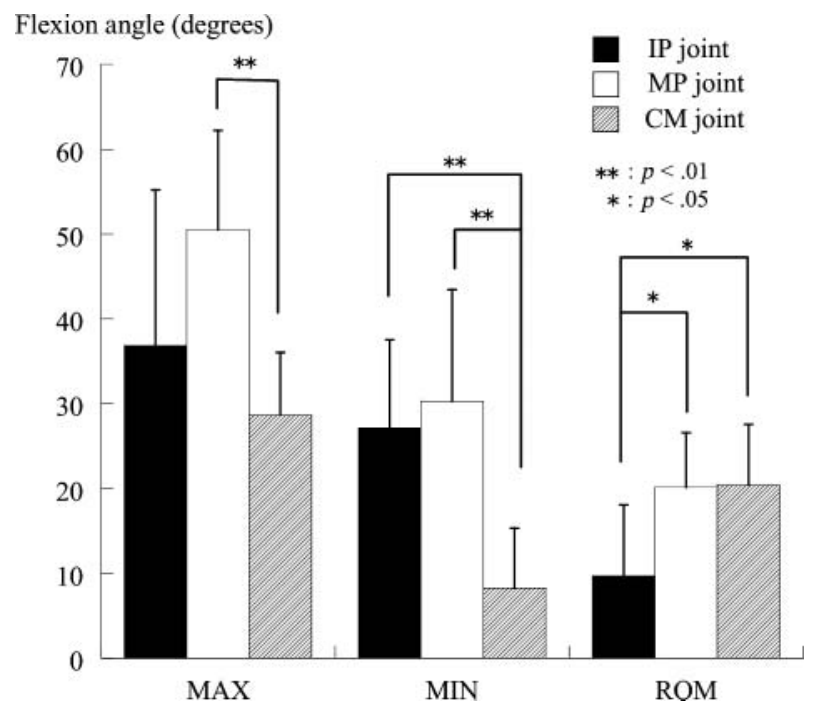

Figure 3. The mean maximum flexion angle of the MP joint was significantly larger than in the $\mathrm{CM}$ joint, and the mean minimum flexion angle of the CM joint was significantly smaller than in the IP and MP joint. The mean range of motion of the IP joint was significantly smaller than in the MP and the CM joint.

\section{Discussion}

The complexity and rapidity of hand motion means it is difficult to make precise measurements for medical evaluation. The thumb especially moves widely in three dimensions when grasping, pinching or performing similar manipulations in our daily environment ( $\mathrm{Su}$ et al. 2003). The structure of the thumb differs from other digits in bone size and in the relation of the proximal phalanx to the carpus (Kaplan and Smith 1984). The function of the thumb also differs from the other fingers because it has a movable

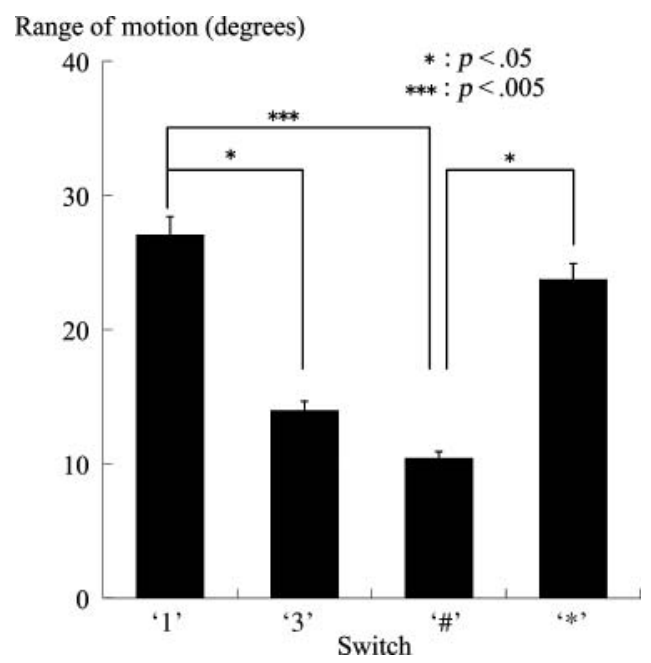

Figure 4. When different key-pushing motions were compared, only the $\mathrm{CM}$ joint showed a significant difference in the range of motion. metacarpal, permitting the special function of opposition, a unique location in front of all the other fingers, in addition to common finger activities. Opposition of the thumb results principally from motion in the first $\mathrm{CM}$ joint, and it also involves a rotary motion of the first metacarpal around its longitudinal axis, but this rotation cannot actively be produced without simultaneous motion about the other axes of the joint.

Most reports of hand motion analysis have involved measurement by electromyography (EMG). Darling and Cole (1990) measured EMGs of the flexor digitorum superficialis, extensor digitorum communis, first dorsal interosseus and lumbrical muscle of the index finger, using fine wires and surface electrodes, during flexion/extension of the index finger. They compared the EMGs and the flexion angle of the MP and PIP joints, concluding that muscle activity is necessary not only to produce joint motion, but also to counteract segmental interaction torques.

Burgar et al. (1997) studied the feasibility of simultaneously measuring maximal voluntary fingertip force generation and EMG activity without significant influence from indwelling fine wire electrodes in all seven muscles of the index finger; they identified skin penetration landmarks, the direction of needle advancement and the depth of muscle fibres.

Li et al. (2000) measured the individual force of the index, middle, ring and little fingers with force sensors, while monitoring the EMG of the extensor digitorum communis, and calculated the finger joint moment from the external and internal forces. They concluded that the joint moment decreased when the point of force application varied in the proximal direction from the distal phalanx to the proximal IP joint.

Some experimental measurements of hand motion, however, have used video-based motion analysis systems. An et al. (1991) developed a 3-D kinematic calculation method based on the output from a video-based passive marker detection system, and found the accuracy to be $150 \mu \mathrm{m}$. The author designed a finger motion analysis study of the professional pianist, in which thumb, index, middle and little finger movements were measured during pianoplaying (Sakai et al. 1996, 2006). The author also studied a finger motion analysis of the myelopathy hand, which is a specific finger motion pattern in patients with cervical myelopathy (Sakai 2005). However, it remains questionable whether the surface markers represent the actual movements of the corresponding underlying bones.

Rash et al. (1999) compared 3-D video motion analysis and 2-D lateral view fluoroscopy, measuring the extension/flexion angles of the DIP, PIP and MCP joints of the index finger. They concluded that the difference between the surface and bone landmarks was no more than 5 degrees during dynamic motion analysis.

Kuo et al. (2003) pointed out that the thumb has a much more complex motion than the other fingers, and they 
investigated the difference between skin surface sensors and bone sensors on the first and third metacarpal bones of human cadavers. Their conclusion was that the angular differences and displacements between the centres of the skin and bone sensors were less than 4.9 degrees and $2.8 \mathrm{~mm}$. Together, these studies suggest that video-based marker detection systems are trustworthy even in the measurement of complex thumb motion.

In the current study, the range of flexion angles showed that the thumb motion on pushing the keys of the cellular phone was produced mainly by the MP and the CM joints. Both the maximum and minimum flexion angles were larger in the MP joint than the CM joint. That is, the range of motion of the CM joint was smaller (from 8.2 to 28.7 degrees, on average) than that of the MP joint (from 30.3 to 50.0 degrees).

On the other hand, the $\mathrm{CM}$ joint showed a larger range of motion on pushing the distant keys of the cellular phone keypad than the MP and IP joints. This result suggests that the ability to reach keys in different areas of the cellular phone keypad is regulated by changing the flexion angle of the $\mathrm{CM}$ joint. It also suggests that the $\mathrm{CM}$ joint controls the basic motion of the thumb according to the key being pushed, and in each position, both the MP and CM joints work together to push the keys of the cellular phone. This fact would prove that de Quervain's disease, tenosynovitis in the abductor and extensor tendon of the thumb, is often seen in the hand problem from cell phone overuse (Yoong, 2005; Storr et al. 2007).

In other words, the $\mathrm{CM}$ joint regulates the distance between the tip of the thumb and the phone key, and the MP joint has the additional regulatory function of allowing the thumb to reach the most distant keys. This result suggests that the distance between the thumb fingertip and each key should be considered in cellular keyboard design, in order to prevent overload in thumb movement.

As the cellular phone is a kind of sophisticated microcomputer, its input motion analysis is important not only for the improvement of cellular phone keypads, but also for the development of next-generation microcomputers.

As the MP joint is 2-D and CM joint is 3-D, the current study has the limitation of concentrating on only the flexion/extension angle, not adduction/abduction and pronation/supination. An additional study with more detailed, 3-D measurement of the thumb joints should be needed in order to clarify the medical problems in hands from cellular phone use.

\section{Acknowledgments}

We would like to thank Professor Sumiko Yamamoto, MD, for her contribution to the study.

\section{References}

An KN, Growney E, Chao EYS. 1991. Measurement of joint kinematics using expertvision system. Biomed Sci Instrum. 27:245-252.

Burgar CG, Valero-Cuevas FJ, Hentz VR. 1997. Fine-wire electromyographic recording during force generation: application to Index Finger Kinesiologic Studies. Am J Phys Med Rehabil 76(6):494-501.

Darling WG, Cole KJ. 1990. Muscle activation patterns and kinetics of human index finger movements. J Neurophysiol. 63(5):1098-1108.

Eliyahu I, Luria R, Hareuveny R, Margaliot M, Meiran N, Shani G. 2006. Effects of radiofrequency radiation emitted by cellular telephone on the cognitive functions of humans. Bioelectromagnetics. 27(2):119-126.

Johal S, Napier F, Britt-Compton J, Marshall T. 2005. Mobile phones and driving. J Public Health (Oxf). 27(1): 112113.

Jokela K, Puranen L, Sihvonen AP. 2004. Assessment of the magnetic field exposure due to the battery current of digital mobile phones. Health Phys. 86(1):56-66.

Kaplan EB, Smith RJ. 1984. Mechanism of action of the fingers, the thumb, and the wrist. In: Spinner M, editor. Kaplan's functional and surgical anatomy of the hand. 3rd ed. Philadelphia (PA): Lippincott Williams \& Wilkins. p. 283352.

Kuo LC, Cooney WP 3rd, Oyama M, Kaufman KR, Su FC, An KN. 2003. Feasibility of using surface markers for assessing motion of the thumb trapeziometacarpal joint. Clin Biomech (Bristol Avon). 18(6):558-563.

Li ZM, Zatsiorsky VM, Latash ML. 2000. Contribution of the extrinsic and intrinsic hand muscles to the moments in finger joints. Clin Biomech (Bristol Avon). 15(3):203211.

Rash GS, Belliappa PP, Wachowiak MP, Somia NN, Gupta A. 1999. A demonstration of validity of 3-D video motion analysis method for measuring finger flexion and extension. $\mathrm{J}$ Biomech. 32(12):1337-1341.

Sakai N. 2005. Finger motion analysis of the patients with cervical myelopathy. Spine, 30(24):2777-2782.

Sakai N, Liu MC, Su FC, Bishop AT, An KN. 1996. Motion analysis of the fingers and wrist of the pianist. Med Probl Perform Art. 11(1):24-29.

Sakai N, Liu MC, Su FC, Bishop AT, An KN. 2006. Hand span and digital motion on the keyboard: concerns of overuse syndrome in musicians. J Hand Surg [Am]. 31(5):830-835.

Storr EF, de Vere Beavis FO, Stringer MD. 2007. Texting tenosynovitis. New New Zealand Med J. 120(1267):107108.

Su FC, Kuo LC, Chiu HY, Chen-Sea MJ. 2003. Video-computer quantitative evaluation of thumb function using workspace of the thumb. J biomech. 36(7):937-942.

Su FC, Kuo LC, Chiu HY, Hsu HY, 2002. The validity of using a video-based motion analysis system for measuring maximal area of fingertip motion and angular variation. Proc Inst Mech Eng [H]. 216(4): 257-263.

White MP, Eiser JR, Harris PR. 2004. Risk perceptions of mobile phone use while driving. Risk Anal. 24(2):323334.

Yoong JK. 2005. Mobile phones can be a pain - text messaging tenosynovitis. Hosp Med. 66(6):370-373. 

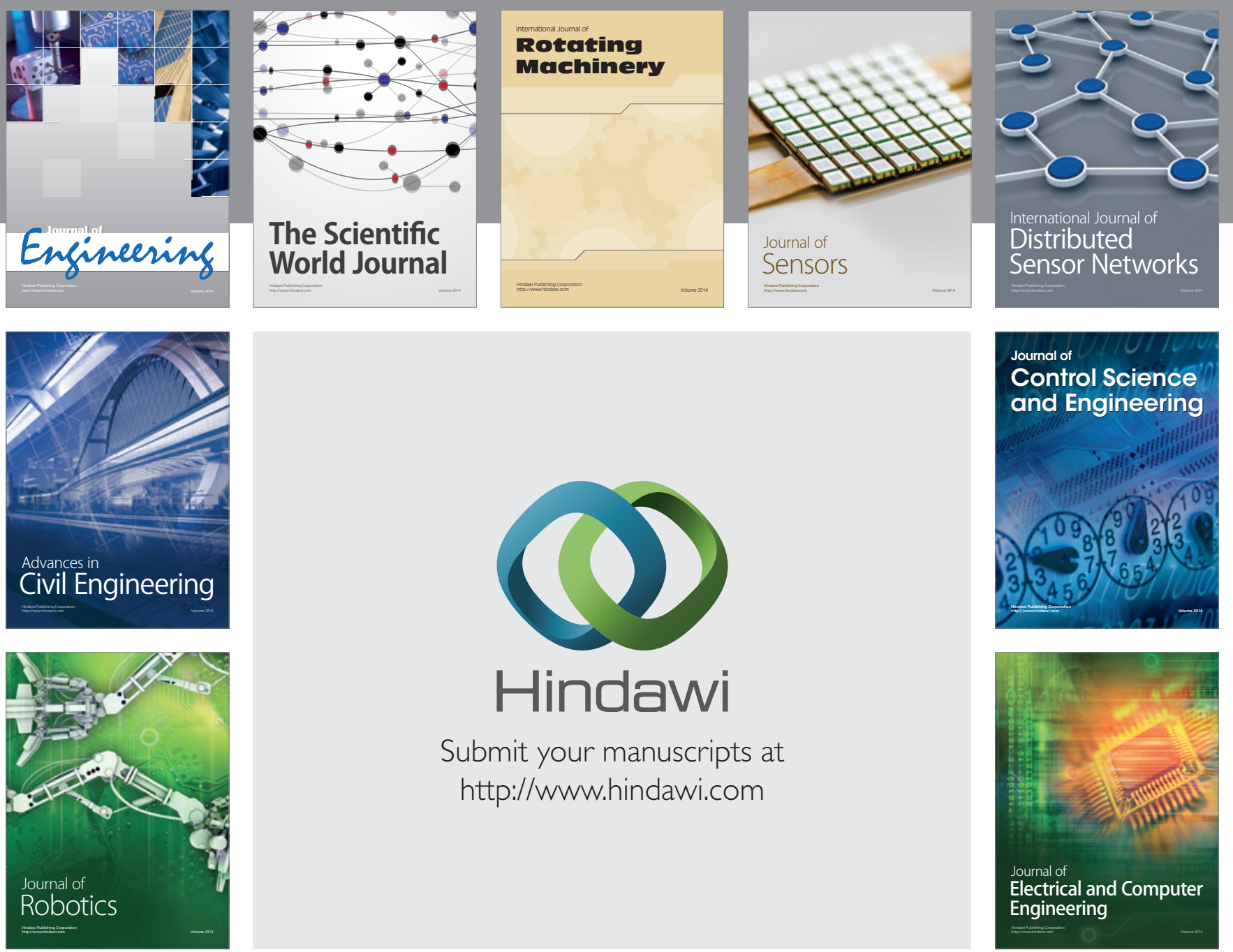

Submit your manuscripts at

http://www.hindawi.com
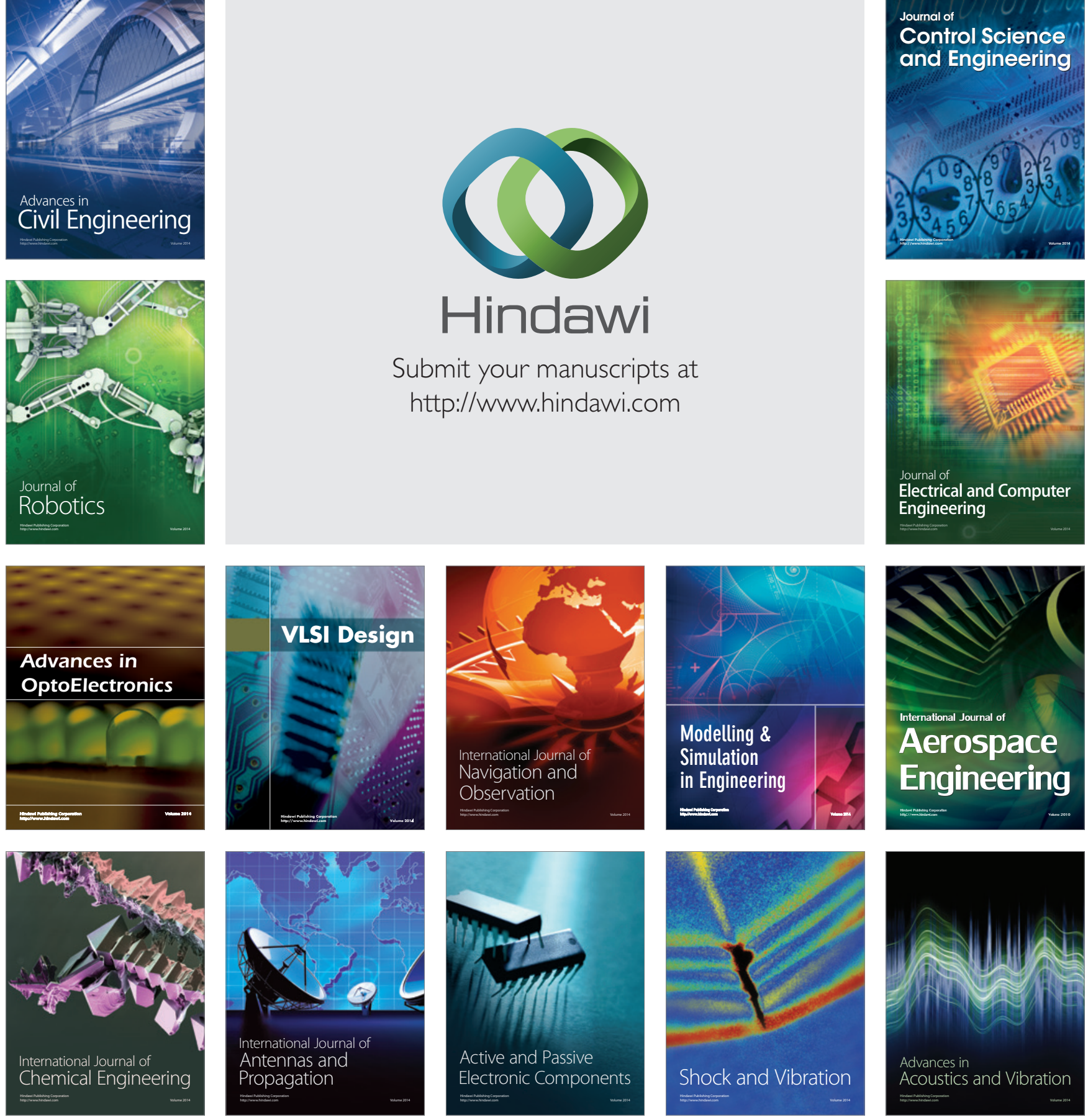